\title{
Uma Análise Preliminar da Aplicação do Método 300 em Turmas de Algoritmos e Programação
}

\author{
Amaury Antônio de Castro Junior ${ }^{1}$, Luciana Montera Cheung ${ }^{1}$, \\ Esteic Janaína Santos Batista ${ }^{3}$, Anderson Corrêa de Lima ${ }^{2}$ \\ ${ }^{1}$ Faculdade de Computação - UFMS \\ Av. Costa e Silva, S/N - Bairro Universitário \\ 79.070-900 - \\ \{amaury.junior, luciana.montera\}@ufms.br \\ ${ }^{2}$ Câmpus de Ponta Porã - UFMS \\ Rua Itibiré Vieira, S/N - Julia Oliveira Cardinal - BR 463 - Km \\ anderson. limadufms.br \\ ${ }^{3} \mathrm{SENAC}$ - MS \\ Rua 26 de Agosto, 835 - Centro - 79.002-081 - Campo Grande - MS \\ esteic.batista@ms.senac.br
}

\begin{abstract}
The challenges in algorithms and programming learning are one of the reasons for the high dropout rates in the early years of computer science courses. This fact motivate the search and the application of new teaching strategies. So, active learning methodologies are presented as an alternative to help teachers in activities that promote greater interaction between students, increase engagement and learning possibilities. This work describes an experience, including actions and observations about application of an active and collaborative learning method, known as Method 300. The method was applied during the first weeks of class of an Algorithms and Programming discipline, in a group of freshmen from a Computer Science course. This document describes application strategies and discusses some preliminary results obtained from the application of this method.
\end{abstract}

Resumo. As dificuldades de aprendizado de algoritmos e programação representam uma das razões para os altos índices de evasão nos primeiros anos dos cursos da área de computação e informática. Tal fato vem motivando a busca e a aplicação de novas estratégias de ensino. Neste cenário, as metodologias ativas de aprendizagem apresentam-se como uma alternativa, auxiliando o professor em atividades que promovam maior interação entre os alunos, aumentando o engajamento e as possibilidades de aprendizagem. Este trabalho apresenta uma análise preliminar sobre a aplicação de um modelo de aprendizagem ativa e colaborativa, conhecido como Método 300. O método foi aplicado durante as primeiras semanas de aula de uma disciplina de algoritmos e programação, em uma turma de ingressantes de um curso de Ciência da Computação. O relato descreve estratégias de aplicação e discute alguns resultados preliminares observados a partir da aplicação deste método. 


\section{Introdução}

As disciplinas ofertadas no primeiro semestre de um curso de nível superior, em geral, oferecem um grande desafio aos professores. Além da grande quantidade de alunos, que é uma realidade nos grandes centros e em cursos de maior procura, há também uma heterogeneidade ocasionada pelas diferenças culturais, de estilos de aprendizagem e de trajetórias de formação distintas [Fragelli 2019].

Depois de atravessarem um grande período de estresse e ansiedade, durante a preparação e realização dos processos seletivos das universidades, os alunos selecionados são colocados diante de um processo de transição para o ensino superior. Esta transição consiste, entre outros fatores, de eventuais afastamentos de familiares e amigos, preocupação com as questões financeiras e de subsistência, além da necessidade de melhor gestão de tempo, compatibilizando as rotinas de estudo com as atividades diárias e tarefas domésticas [Hoed 2016]. Nesse contexto, não é incomum encontrar alunos com sinais de ansiedade, como crises de choro, alterações do padrão de sono, nervosismo constante, dificuldade em se concentrar, entre outros aspectos emocionais que provocam impacto no seu desempenho no curso.

No caso dos cursos superiores da área de computação, a situação não é diferente. No entanto, existem outras causas associadas à evasão que têm relação com as estruturas curriculares desses cursos. Em geral, os primeiros períodos dos cursos superiores da área de computação possuem disciplinas voltadas para o uso de algoritmos e programação. Tais conteúdos servem de base para que o aluno aprenda a desenvolver programas de computador. Portanto, devido à relevância para a formação superior de um profissional da área de computação, cabe também considerar que o insucesso nessas disciplinas pode contribuir para o fenômeno da ansiedade e, em alguns casos, resultando na evasão [Hoed 2016].

Carvalho et al. [Carvalho et al. 2019] constataram que o desempenho acadêmico no primeiro semestre do curso é um dos fatores de maior impacto para a evasão do aluno ou para o seu engajamento definitivo no curso. No entanto, como o desempenho só pode ser avaliado após o término do primeiro semestre, foram analisados os aspectos que pudessem ser considerados já nas primeiras semanas de aula. Foram observados dois fatores como principais indicadores de impacto no coeficiente de rendimento no primeiro período letivo:

1. A participação em atividades de acolhimento de calouros.

2. Experiência prévia do estudante com linguagens de programação.

Diante dessa análise prévia, é possível inferir que os aspectos emocionais (acolhimento) e técnicos (conhecimento prévio) estão entre os fatores significativos a serem considerados nas abordagens pedagógicas aplicadas nos períodos iniciais do curso. Portanto, o uso de métodos que promovam engajamento do aluno no estudo de conteúdos técnicos, por meio de um ambiente colaborativo, interativo e acolhedor podem representar uma alternativa promissora e com bons resultados para melhorar o desempenho dos ingressantes em disciplinas introdutórias do curso.

Mesmo diante de todo o esforço das universidades em ações de acolhimento dos novos alunos, o papel do professor é fundamental no processo de acomodação e motivação do estudante no início do curso. O uso e aplicação de métodos e estratégias inovadoras de 
ensino apresenta-se como uma alternativa para promover o engajamento e desenvolver o interesse e a motivação dos alunos pela sua própria aprendizagem.

Neste trabalho, é apresentada uma estratégia de aplicação de uma metodologia ativa de aprendizagem, denominada Método 300, proposto por, proposto por [Fragelli and Fragelli 2016]. A estratégia foi adotada em uma disciplina de Algoritmos e Programação ofertada para uma turma de ingressantes do Curso de Ciência da Computação da Faculdade de Computação da Universidade Federal de Mato Grosso do Sul (FACOM/UFMS).

Este trabalho está organizado em seções, sendo esta a Introdução. Na Seção 2 é apresentado o conceito de metodologias ativas e seus benefícios no ensino de programação. Na Seção 3 é apresentado o Método 300 desde a sua concepção até o seu formato de aplicação. A Seção 4 apresenta um relato da aplicação do Método 300 na disciplina de Algoritmos e Programação, ofertada para uma turma de ingressante de um curso de Ciência de Computação. Por fim, na Seção 5 são feitas as considerações finais e brevemente discutidos os resultados preliminares obtidos da aplicação do método, com a apresentação de perspectivas e possíveis estratégias de aplicação e pesquisas futuras.

\section{Metodologias Ativas no Ensino de Programação}

As metodologias ativas de aprendizagem e suas estratégias de aplicação têm se mostrado uma boa alternativa para estimular o protagonismo dos alunos no processo de ensino e de aprendizagem, além de fortalecer o engajamento em sala de aula e em outros ambientes educacionais não-formais. Em suas premissas e organização, se relacionam com teorias de concepções de aprendizagem interacionistas que defendem que o desenvolvimento humano e a aquisição de conhecimento como um resultado de ação recíproca do homem com o meio e o organismo [Davis and Oliveira 1994].

No que se refere à aplicação de metodologias ativas em disciplinas introdutórias de programação, vários trabalhos, com resultados relevantes foram desenvolvidos nos últimos anos [Bigolin et al. 2020, Mourão 2017, Freire et al. 2019, Diemer et al. 2020, Zacarias and Mello 2019].

Em [Bigolin et al. 2020], os autores apresentam um relato de experiência sobre a aplicação das metodologias ativas em disciplinas de programação e estruturas de dados, usando uma estratégia de aprendizagem baseada em problemas (no inglês, Problem Based Learning - PBL). Os gráficos e tabelas percentuais apresentados no trabalho comprovam os bons resultados obtidos a partir da aplicação do método em uma turma de um curso de Sistemas de Informação, com um grande número de alunos nas disciplinas introdutórias de programação. A aplicação das metodologias ativas melhoraram as taxas de sucesso e reduziram a evasão nas disciplinas introdutórias, além de motivar a realização de workshops de qualificação docente, com debates, discussões e troca de ideias entre docentes, visando ao compartilhamento de experiências e as melhorias em suas práticas pedagógicas.

Em [Mourão 2017], a autora descreve a adoção de uma estratégias de aprendizagem baseada em problemas com o método de Dojô de Programação (no inglês, Coding Dojo). Este método de ensino é baseado nas técnicas de artes marciais japonesas, onde o dojô é o espaço utilizado pelos lutadores para a realização de seus treinos e testes para 
mudança de nível. No caso do dojô de programação, trata-se de locais onde os estudantes se reúnem para competições baseadas em desafios, da mesma forma como é feito em treinos e provas de artes marciais. A ideia, além de tornar a prática mais divertida é a de promover um ambiente inclusivo, cooperativo e colaborativo, baseado na troca de experiências e no networking entre os participantes. O método foi aplicado em disciplinas de Lógica de Programação, em turmas de computação de duas instituições de ensino superior, sendo uma pública e outra particular. Os resultados demonstram um aumento significativo e gradativo da taxa de sucesso dos alunos na disciplina.

Em [Diemer et al. 2020], os autores aplicaram a estratégia de Aprendizagem por Pares (no inglês, Peer Instruction). Esta metodologia ativa é caracterizada pelo trabalho em equipes, baseando-se na troca de experiências e conhecimento entre os estudantes, estimulada pelo docente, de tal forma que as equipes de dois ou mais alunos atuem colaborativamente na resolução de um problema, explorando conteúdos e melhorando suas habilidades por meio da cooperação. Os autores realizaram estudos comparativos entre alunos de uma turma da disciplina de Algoritmos e Programação onde a metodologia foi aplicada com os alunos de outra turma onde foram aplicadas metodologias convencionais. Os resultados apresentados pelos autores apontam uma grande contribuição da estratégia Peer Instruction para a construção coletiva de significados, levando a maioria dos estudantes para o ponto de chegada mais próximo do objetivo pedagógico previamente definido.

Em [Freire et al. 2019], os autores relatam a experiência da aplicação de encontros de tutoria, baseados em metodologias ativas, para o ensino introdutório de programação. O método foi aplicado por meio do planejamento de 16 (dezesseis) encontros de tutoria, com conteúdos previamente definidos e coordenados por docentes, mas com a gerência e a atuação de alunos como tutores. A organização da atividade é baseada no que acontece na Educação à Distância $(\mathrm{EaD})$, com um sistema de tutoria hierárquico e caracterizado por um ou mais alunos-tutores que oferecem suporte individualizado aos colegas com maior dificuldade. Os resultados apontam uma boa aceitação do método por parte dos estudantes, apesar de alguns problemas identificados como a incompatibilidade de horários entre o tutor e seus tutorados, além do pouco engajamento dos estudantes dos primeiros períodos do curso.

Por fim, em [Zacarias and Mello 2019], é apresentada uma revisão sistemática sobre metodologias de ensino de lógica de programação e algoritmos em cursos de graduação. Os autores discutem métodos e ferramentas identificados a partir de um levantamento bibliométrico no Google Acadêmico®. O trabalho apresentou e discutiu 10 (dez) artigos científicos que trataram de abordagens pedagógicas inovadoras com aplicações bem sucedidas.

Diante das referências apresentadas, é possível identificar a necessidade de inovação dos métodos de ensino de algoritmos e programação, em especial, nas turmas de ingressantes que, geralmente, são numerosas e com maior heterogeneidade. Nesse contexto, a seção seguinte apresenta o Método 300, criado por um professor brasileiro e caracterizado como uma metodologia ativa inovadora, que também incentiva a cooperação e favorece o engajamento dos alunos por meio de uma estratégia de avaliação inteligente e colaborativa. 


\section{O Método 300}

O Método 300 foi desenvolvido pelo Prof. Ricardo Fragelli [Fragelli and Fragelli 2016, Fragelli 2019, Fragelli and Fragelli 2017], da Universidade de Brasília (UnB), inspirado no filme "Trezentos", que retrata a Batalha de Termópilas, conhecida como a Batalha dos 300. Nessa batalha, o exército Espartano, bem menos numeroso e liderado pelo rei Leônidas, conseguiu enfraquecer o gigantesco exército persa por meio de um trabalho de coletividade, onde os soldados atuavam como uma unidade, defendendo uns aos outros durante a batalha.

Este método pode ser definido como uma estratégia inovadora de aprendizagem ativa, reunindo características das estratégias de aprendizagem por pares e tutoria, que são muito utilizadas na aprendizagem ativa, como vimos na Seção 2. De forma resumida, o método consiste da prática de aprender ensinando, permitindo que os alunos com melhor desempenho sejam mentores de seus colegas de turma, incentivando a empatia e a cooperação entre estudantes que estão, momentaneamente, em estágios de aprendizagem distintos. Para identificar os estudantes e seus respectivos estágios de aprendizagem, todos participam de uma avaliação individual e, os grupos de colaboração são construídos com base no rendimento de cada um nesta avaliação.

Por exemplo, para a formação de $n$ grupos de colaboração de estudantes, devemos seguir os seguintes passos, com base no resultado da avaliação individual realizada:

1. os estudantes são ordenados pela nota da avaliação individual, da maior para a menor;

2. atribui-se um número a cada estudante, iniciando de 1 a $n$ depois repete-se o processo de numeração de $n$ até 1 , até que todos os estudantes tenham um número atribuído a eles;

3. os estudantes com o mesmo número farão parte de um mesmo grupo.

Deve-se observar que o valor de $n$ tem impacto no tamanho dos grupos e pode ser definido pelo professor, de acordo com o número da estudantes da turma. Assim, da forma como é feita a distribuição dos grupos, garante-se que todos serão compostos por estudantes de diferentes estágios de aprendizagem e/ou conhecimento prévio, garantindo a heterogeneidade. O objetivo de cada grupo é o de dar apoio aos estudantes com baixo rendimento, por meio de encontros presenciais ou outros meios de comunicação e interação. A Figura 1 ilustra um fluxograma com cada uma das etapas de aplicação do Método 300.

Sendo assim, um estudante que teve um baixo rendimento na primeira prova poderá realizar uma nova avaliação do conteúdo, desde que cumpra algumas metas com o apoio do grupo. As metas devem ser pensadas pelo docente de modo a estimular a interação dos estudantes com o conteúdo e, em encontros presenciais, oportunizar a troca de saberes.

Em [Fragelli and Fragelli 2016], os autores descrevem a aplicação do Método 300 em cursos da área de saúde. Na seção seguinte, é feito um relato da aplicação do Método 300 em uma turma de ingressantes do Curso de Ciência da Computação da FACOM/UFMS durante o primeiro período letivo de 2020.

O Método 300, em particular, se relacionam com a teoria Vygostskyana de aprendizagem, que define a Zona de Desenvolvimento Proximal (ZDP), que seria a distância 


\section{Método 300}

\section{Passo-a-passo da aplicação}

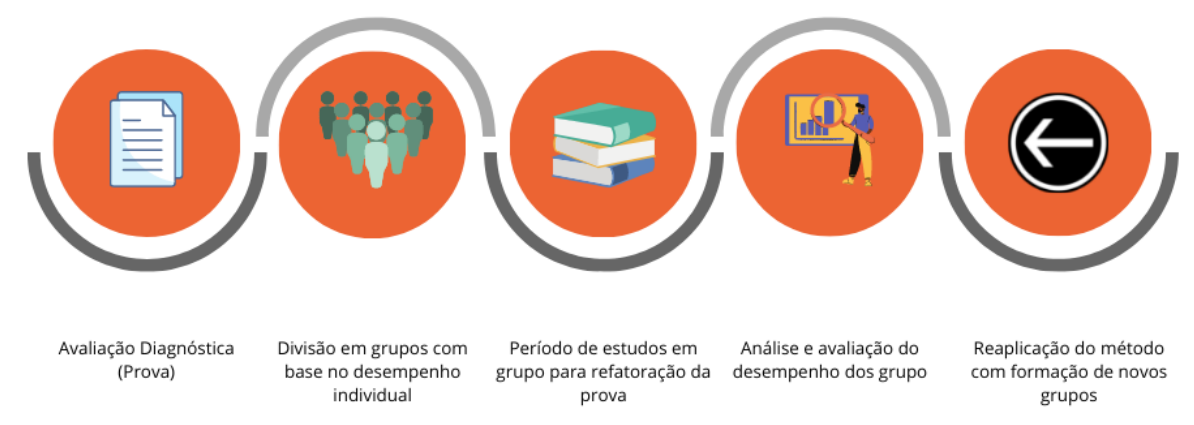

Figura 1. Fluxograma que representa o passo-a-passo da aplicação do Método 300.

entre o nível de desenvolvimento real, que é definido com os problemas ou desafios que uma o aprendiz consegue resolver de forma independente; e o nível de desenvolvimento potencial, que diz respeito ao que ela consegue resolver sob orientação de um tutor ou em colaboração com outros colegas. Desta forma, aprendizagem e desenvolvimento se desenvolvem em níveis diferentes mais um influencia o outro. No Método 300, a ZPD é evidenciada a partir dos desafios que os alunos devem realizar em colaboração um com os outros, por meio do papel de tutoria daqueles que, em um determinado momento, possuem mais facilidade em determinado conteúdo. O professor, nesse caso, é um facilitador e mediador do conhecimento, responsável por propor desafios aos estudantes e acompanhar o desenvolvimento das equipes.

Desta forma, o método permite que os estudantes desenvolvam uma postura mais sensível em relação ao outro, avaliando com maior generosidade as dúvidas dos colegas, evidenciando uma metodologia ativa e colaborativa de aprendizagem, que requer a ação dos estudantes no seu processo de aprendizagem [Borges and Sbardelotto 2017].

\section{Um Exemplo de Aplicação no Ensino de Algoritmos e Programação}

As disciplinas de Algoritmos e Programação ofertadas para os primeiros períodos dos cursos da área de computação da FACOM/UFMS caracterizam-se, em geral, por turmas numerosas, entre 40 e 80 alunos matriculados e por altos índices de evasão e reprovação.

Dessa forma, a busca por estratégias inovadoras e que possam melhorar esse cenário é fundamental. Além disso, o compartilhamento dessas estratégias entre os docentes e pesquisadores da área pode influenciar a sua adoção em outras disciplinas dos 
cursos.

As turmas de ingressantes em cursos de computação são, de forma geral, muito heterogêneas. Com a criação das escolas técnicas e institutos federais, muitos estudantes já chegam ao ensino superior conhecendo os fundamentos de algoritmos e com domínio de uma ou mais linguagens de programação, enquanto outros, provenientes do ensino médio regular, nunca tiveram contato com programação. Como se não bastassem as diferenças de conhecimento prévio adquiridas, os estudantes acabam se organizando de forma a acentuar essas diferenças, relacionando-se com os colegas que já conheciam ou que possuem o mesmo nível de conhecimento e interesses comuns.

Com o Método 300, foi possível reduzir essa distância, aproximando alunos que, possivelmente, jamais teriam uma relação de cooperação espontânea. Para isso, foi utilizado como ferramenta auxiliar de avaliação, o URI ${ }^{1}$, por meio de uma disciplina criada pelo professor e por maratonas de programação propostas como forma de avaliação individual e por equipes. O método foi aplicado em uma turma de ingressantes, durante o semestre de 2020-1.

Seguindo o que é proposto pelo Método 300, após a avaliação, foi realizada a distribuição dos grupos, que receberam nomes de personagens do filme "Star Wars". Os grupos reuniram-se durante as aulas e discutiram as soluções dos problemas propostos e os conceitos envolvidos, sob a orientação do professor. Foram realizadas duas maratonas internas de programação entre os estudantes da turma. A primeira delas como avaliação individual diagnóstica, com o objetivo de formar os grupos. A segunda foi realizada após a aplicação do Método 300, depois do período de atividades e metas estabelecidas pelos grupos criados.

Os resultados da primeira maratona (avaliação individual) e da segunda maratona (após a aplicação do método) são mostrados na Figura 2 (a) e (b), respectivamente.

Os balões coloridos da Figura 2 representam problemas que foram resolvidos. Os nomes dos alunos foram omitidos por questões de privacidade. São apresentados apenas os resultados e fica evidente que o Método 300 contribuiu com a formação de uma unidade na turma de algoritmos e programação, fazendo com que a maioria dos estudantes pudesse atingir um nível de conhecimento mais próximo entre si, reduzindo a heterogeneidade.

Além dos impactos no desenvolvimento mais uniforme da turma, deve-se destacar a melhoria da empatia e da integração entre os estudantes.

\section{Considerações Finais}

Infelizmente, em razão da pandemia e da transição para o ensino remoto, não foi possível o aprofundamento dos resultados de aplicação do método que tinham, inicialmente, sido planejados para o ensino presencial. No entanto, o desempenho da turma na disciplina e no período de aplicação do Método 300 mostram-se promissores. Comparando as médias gerais das turmas e as taxas de sucesso e de reprovação dos estudantes que cursaram a disciplina de algoritmos e programação I nos últimos 6 (seis) semestre letivos, é possível perceber o impacto da aplicação do método na turma ofertada no semestre de 2020-1.

Na Tabela 1 observa-se que, no período de aplicação do Método 300, os resulta-

\footnotetext{
${ }^{1}$ https://www.urionlinejudge.com.br
} 


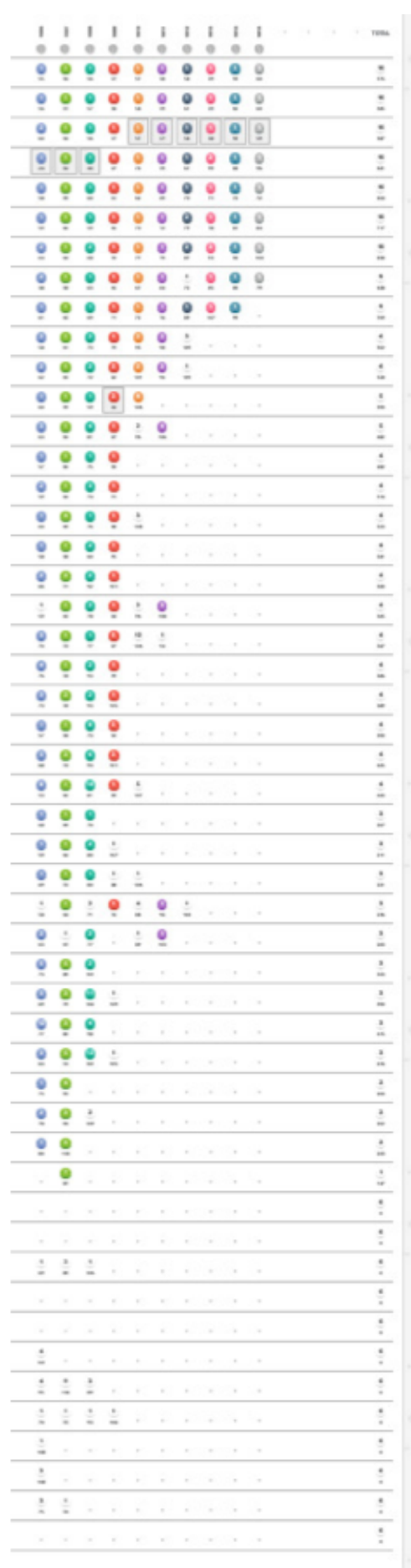

(a)

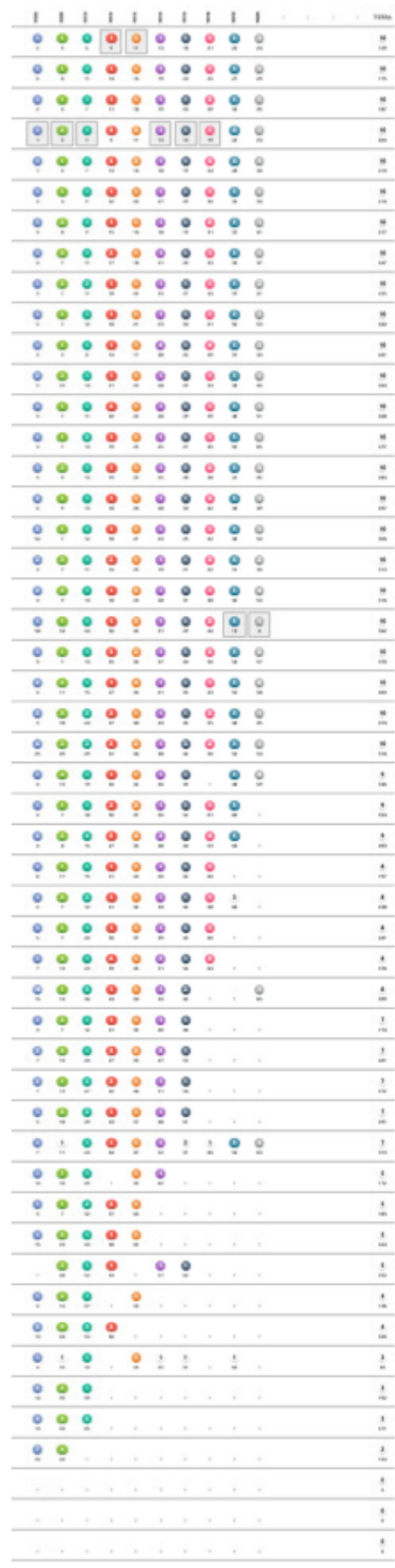

(b)

Figura 2. (a) Desempenho dos alunos na avaliação individual ANTES da aplicação do método. (b) Desempenho dos alunos na avaliação individual APÓS a aplicação do método.

dos foram os melhores entre os 6 (seis) períodos de oferta da disciplina. Isso demonstra o potencial do método para promover maior engajamento e desenvolvimento uniforme da turma. Entretanto, ainda existem outros fatores a serem analisados, tais como a permanência no curso. Tais fatores serão objetos de análises futuras. 
Tabela 1. Dados obtidos do Sistema Acadêmica da universidade, contendo as informações sobre as disciplinas de Algoritmos e Programação I ofertadas nos períodos de 2018-1 a 2020-2. Em destaque, o período em que foi aplicado o Método 300.

\begin{tabular}{|c|c|c|c|c|}
\hline Semestre & Matriculados & \% Aprovados & \% Reprovados & Média da Turma \\
\hline $2018-1$ & 86 & $29,1 \%$ & $70,9 \%$ & 3,3 \\
\hline $2018-2$ & 46 & $54,3 \%$ & $45,7 \%$ & 4,8 \\
\hline $2019-1$ & 67 & $50,7 \%$ & $49,3 \%$ & 4,8 \\
\hline $2019-2$ & 23 & $17,4 \%$ & $82,6 \%$ & 2,5 \\
\hline $\mathbf{2 0 2 0}-1$ & $\mathbf{6 5}$ & $\mathbf{6 1 , 5 \%}$ & $\mathbf{3 8 , 5 \%}$ & $\mathbf{5 , 4}$ \\
\hline $2020-2$ & 23 & $21,7 \%$ & $78,3 \%$ & 2,3 \\
\hline
\end{tabular}

Obviamente, muitos fatores ainda devem ser analisados para validação dos impactos e resultados efetivos provenientes da aplicação do Método 300. No entanto, o que percebe-se é um maior engajamento e comprometimento dos estudantes com o desenvolvimento próprio e dos colegas de turma. Tais impactos são percebidos a partir de atitudes e posturas dos próprios estudantes, no apoio e incentivo aos colegas de turma.

Em 2021, a estratégia foi adaptada para o ensino remoto de emergência na universidade e está em fase de aplicação na turma de ingressantes deste ano. Foram escolhidas duas turmas da disciplina de Algoritmos e Programação, sendo uma do Curso de Ciência da Computação e outra do Curso de Engenharia de Software. Os resultados obtidos serão analisados e organizados na forma de gráficos e tabelas e relatados em trabalhos futuros. Também será possível comparar as taxas de sucesso ao longo do tempo.

Constata-se, a partir desta análise inicial, que é possível ampliar o engajamento e o interesse dos estudantes pela disciplina de Algoritmos e Programação, mesmo dos que não possuem conhecimento prévio, por meio da aplicação de métodos inovadores e que promovam a coesão e a cooperação entre os colegas de uma mesma turma. Além disso, é possível melhorar os resultados a partir do aumento da população de alunos, por meio de uma ação conjunta, envolvendo os docentes responsáveis pelas disciplinas de algoritmos e programação introdutórias, o que também viabilizará a disseminação do método como alternativa nos anos iniciais de todos os cursos de computação da universidade.

\section{Referências}

Bigolin, N. M., Silveira, S. R., Bertolini, C., de Almeida, I. C., Geller, M., Parreira, F. J., da Cunha, G. B., and Macedo, R. T. (2020). Metodologias ativas de aprendizagem: um relato de experiência nas disciplinas de programação e estrutura de dados. Research, Society and Development, 9(1):5.

Borges, L. F. P. and Sbardelotto, V. S. (2017). O ensino na educação superior: uma análise da metodologia "trezentos" e sua relação com a pedagogia histórico-crítica. Revista Internacional de Educação Superior, 3(3):468-477.

Carvalho, L., Santos, A., Nakamura, F., and Oliveira, E. (2019). Detecção precoce de evasão em cursos de graduação presencial em computação: um estudo preliminar. In Anais do XXVII Workshop sobre Educação em Computação, pages 233-243, Belém/PA. SBC, WEI/CSBC. 
Davis, C. and Oliveira, Z. d. (1994). Psicologia na educação. Cortez.

Diemer, M. H., Bercht, M., do Canto Filho, A. B., and Schorr, M. C. (2020). Metodologias ativas no ensino de algoritmos e programação: um relato de aplicação da metodologia peer instruction. Revista Destaques Acadêmicos, 11(4):240-255.

Fragelli, R. (2019). Método Trezentos: Aprendizagem Ativa e Colaborativa, para Além do Conteúdo. Desafios da Educação. Penso, Porto Alegre, 1 edition.

Fragelli, R. R. and Fragelli, T. B. O. (2017). Trezentos: a dimensão humana do método. Educar em Revista, 1(63):253-265.

Fragelli, T. B. O. and Fragelli, R. R. (2016). Uma experiência de aplicação do método trezentos na área da saúde. Educação, Ciência e Saúde, 3(1):10.

Freire, L., Coutinho, J., Lima, V., and Lima, N. (2019). Uma proposta de encontros de tutoria baseada em metodologias ativas para disciplinas de programação introdutória. In Anais dos Workshops do Congresso Brasileiro de Informática na Educação, volume 8, pages 298-307, Brasília/DF. CBIE/SBC.

Hoed, R. M. (2016). Análise da evasão em cursos superiores: o caso da evasão em cursos superiores da área de Computação. $\mathrm{PhD}$ thesis, Universidade de Brasília.

Mourão, A. (2017). Uma proposta da eficiência do uso da metodologia ativa baseada em problemas, utilizando dojo de programação, aplicada na disciplina de lógica de programação. In Anais do Workshop de Informática na Escola, volume 23, pages 667-676, Recife/PE. WIE/CBIE/SBC.

Zacarias, R. O. and Mello, D. R. B. (2019). Metodologias de ensino de lógica de programação e algoritmos em cursos de graduação. Revista Interdisciplinar Pensamento Científico, 5(2):29-44. 